\title{
Competencias genéricas y estrés académico en estudiantes universitarios
}

\author{
Shirlay Lis Llanos Eusebio \\ Universidad Nacional de Educación Enrique Guzmán y Valle
}

\section{Resumen:}

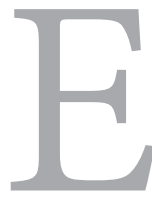

1 objetivo del trabajo fue establecer la relación entre las competencias genéricas y el nivel de estrés en estudiantes universitarios. Se reporta un estudio descriptivo correlacional. La muestra estuvo constituida por 125 estudiantes de ambos sexos pertenecientes a la facultad de Ciencias Sociales y Humanidades de la Universidad Nacional de Educación "Enrique Guzmán y Valle", a los cuales se les aplicó el cuestionario de competencias genéricas de Solanes, Núñez y Rodríguez(2007) y el inventario de estrés académico (I.E.A. Hernández, Polo y Pozo, 1996). A partir del análisis de los resultados se llegó a la siguiente conclusión: El grado de correlación entre las variables competencias genéricas y estrés académico es negativa media, es decir, a mayor competencia genérica menor nivel de estrés académico y a menor competencia genérica mayor nivel de estrés académico.

Palabras clave: competencias, competencias genéricas, estrés, estrés académico.

\section{Summary:}

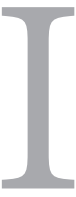
$\mathrm{n}$ this research, a descriptive correlational study is reported. The objective was to establish the relationship between the generic competencies and the level of stress in college students. The sample consisted of 125 male and female students of belonging to the department academy of the School of Social Sciences, National University of Education "Enrique Guzman and Valle", to which we applied the questionnaire generic competencies of Solanes, Nuñez and Rodriguez (2007) and the inventory of academic stress (IEA Hernández, Polo and Pozo, 1996). From the analysis of the results was reached the following conclusion. The degree of correlation between variables generic skills and academic stress is negative average, and more generic skills lower level of academic stress and less generic higher level of academic stress competition.

Keywords: competencies, generic skills, stress, stress level. 


\section{Artículos Empíricos}

\section{Introducción}

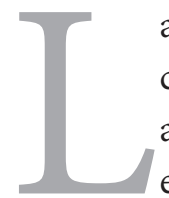

as competencias son conjuntos de conocimientos, procedimientos $\mathrm{y}$ actitudes combinados, coordinados e integrados para realizar alguna acción, se adquieren durante el desarrollo de nuestra experiencia (formativa y no formativa) $y$ permite resolver problemas específicos de forma estratégica, autónoma y flexible. Esta definición es relevante para la formación de los estudiantes en cualquier nivel, por ello, es importante que se investigue en los centros educativos y en el nivel universitario.

En contextos diversos e impredecibles, es sumamente importante conocer las competencias que tienen los estudiantes universitarios, nos encontramos inmersos en la sociedad del conocimiento y una de las características es que la información se crea rápidamente y queda obsoleta también. Cornella (2010) alerta sobre los riesgos de la información en la actualidad; hay un exceso de información que muchas veces acaba por "intoxicarnos". Es necesario buscar la información pertinente, seleccionarla (de entre un abanico de posibilidades), procesarla, tratarla, interpretarla y apropiarse de ella para generar el conocimiento que nos permita resolver las situaciones que se presenten. Es por eso, que los diseños por competencias desarrollan capacidades amplias, que permitan aprender y desaprender para adecuarse a situaciones cambiantes.
Necesitamos conocimientos, habilidades y actitudes imprescindibles en la vida.

Otra característica de esta sociedad, es la complejidad del conocimiento que obedece a una lógica posmoderna que cuesta articular, porque se equipara complejidad y complicación. Las clásicas divisiones entre asignaturas o las clasificaciones de los saberes parecen no servir en el contexto actual. Hay que apostar por un conocimiento integrado que, como sugiere Morin (2001), supere la superespecialización y el reduccionismo. En consecuencia, las competencias genéricas de las personas, proporcionan la movilización de conocimientos y su combinación para responder a situaciones en contextos diversos.

Finalmente, una formación integral que permite a las personas enfrentarse a una sociedad incierta, sociedad red y primacía de las nuevas tecnologías de información y comunicación (NTIC); es una sociedad del bienestar, pero con riesgo de un creciente consumismo compulsivo.

En este sentido, las propuestas por competencias incluyen conocimientos, habilidades y actitudes diferentes, incorporando talentos o inteligencias que tradicionalmente desde los sistemas educativos no se tenían presentes. Hace más de una década, Gardner (1994) mostró la importancia de las inteligencias múltiples. Actualmente, con los diseños por 


\section{Artículos Empíricos}

competencias, todas esas propuestas tienen mayor relevancia que pueden ayudarnos a dar respuesta a una situación de forma eficiente o a adaptarnos a realidades cambiantes.

Lo que se pretende con el desarrollo de competencias genéricas en los estudiantes, es que desde las instituciones de educación superior o lo que fuere, tendrán que promover un núcleo irreductible de conocimientos y destrezas que todo estudiante debiera dominar, por ejemplo, las capacidades de comunicación, el razonamiento científico, la toma de decisiones y, sobre todo, su desarrollo personal ético.

Uno de los retos más relevantes de los estudiantes universitarios es la competitividad, ya sea por un buen rendimiento o por salir al mercado laboral. Son muchos los estudiantes y no tantas las oportunidades del mercado laboral. Ello hace que ser "de los mejores" sea un objetivo que los estudiantes (sobre todo los de los últimos cursos) se plantean por encima incluso de la adquisición de conocimientos. La exigencia académica y los hábitos de trabajo adquiridos en los escalones más bajos del sistema educativo no siempre ayudan a la adaptación natural del estudiante al ámbito universitario.

Todos estos fenómenos acarrean un alto nivel de estrés en los estudiantes. El estrés académico se produce en el ámbito educativo, que podría afectar tanto a profesores como a estudiantes, en cualquier nivel educativo. No obstante, nos centramos en el estrés académico de los estudiantes universitarios, de aquellas situaciones que se producen en mayor medida e intensidad.

Fisher $(1984,1986)$ considera que el ingreso a la Universidad representa un conjunto de situaciones altamente estresantes debido a que el individuo puede experimentar, aunque solo sea transitoriamente, una falta de control del nuevo ambiente, potencialmente generador de estrés y del fracaso académico. Por otro lado, el estilo de vida de los estudiantes se ve modificado según se acerca el período de exámenes, convirtiéndose así sus hábitos en insalubres -exceso en el consumo de cafeína, tabaco, sustancias psicoactivas como excitantes e, incluso, en algunos casos, ingestión de tranquilizantes-, lo que, a la larga, puede llevar a la aparición de trastornos de salud (Hernández, Pozo y Polo, 1994).

Sin embargo, no todos los estudiantes universitarios padecen estrés. La respuesta depende de la disposición personal para hacer frente a las demandas. Afirmar que un estudiante universitario padece estrés o no, resulta de una utilidad cuestionable por su generalidad. Nuestro objetivo es determinar la relación entre las competencias genéricas y el nivel de estrés. Por ello, la importancia de este estudio, la cual tiene por objetivo, analizar y explicar la relación que existe entre estas variables. 


\section{Método}

Participantes

En el estudio participaron un total de 125 estudiantes de ambos sexos pertenecientes al departamento académico de Ciencias Sociales de la Universidad Nacional de Educación "Enrique Guzmán y Valle”.

\section{Materiales}

Se utilizaron dos cuestionarios, unos sobre competencias genéricas de Solanes, Núñez y Rodríguez (2007) de 52 ítems para la evaluación de potenciales constaba de dos ítems de respuesta abierta y cincuenta con formato tipo Likert con seis categorías de respuesta: (1) Siempre, (2) Muchísimas veces, (3) Con frecuencia, (4) Ordinariamente, (5) Ocasionalmente y (6) Casi nunca. El instrumento está dividido en tres dimensiones, las cuales se mencionan en la siguiente tabla.

\begin{tabular}{|c|c|c|c|}
\hline Factor & $\begin{array}{c}\% \text { de varianza } \\
\text { explicada }\end{array}$ & $\begin{array}{c}\text { Consistencia } \\
\text { interna }\end{array}$ & Items \\
\hline \multicolumn{4}{|c|}{ COMPETENCIAS INSTRUMENTALES relacionadas con: } \\
\hline Desempeño del trabajo & $10 ’ 95$ & $0 ’ 81$ & $3-16-18-19-39-42-45-47-48-49-50-51$ \\
\hline Habilidades para la gestión & 9’82 & $0 ’ 79$ & $9-10-20-21-22-38-41$ \\
\hline \multicolumn{4}{|c|}{ COMPETENCIAS SISTÉMICAS relacionadas con: } \\
\hline Liderazgo & 9’38 & $0 ’ 84$ & $11-13-14-15-17-27-28-30-32-34$ \\
\hline Motivación por el trabajo & $7^{\prime} 22$ & $0 ’ 73$ & $1-2-8-12-36-40-44$ \\
\hline Capacidad de aprendizaje & 6’92 & 0’69 & 4-26-37-46 \\
\hline \multicolumn{4}{|c|}{ COMPETENCIAS INTERPERSONALES relacionadas con: } \\
\hline $\begin{array}{l}\text { Relaciones interpersonales } \\
\text { y trabajo en equipo }\end{array}$ & $8^{\prime} 86$ & 0’81 & $23-24-25-29-33$ \\
\hline Total Cuestionario & $53 ’ 15$ & $0 ’ 92$ & 45 \\
\hline
\end{tabular}

También se utilizó el Inventario de Estrés Académico (I.E.A.; Hernández, Polo y Pozo, 1996) el mismo que ha sido diseñado específicamente para la evaluación del estrés académico en universitarios. En esta escala se evalúa once situaciones potencialmente generadoras de estrés en los estudiantes dentro del ámbito académico, en una escala tipo Likert de 1 a 5, (donde 1 representa Nada de estrés y 5 Mucho estrés). 


\section{Artículos Empíricos}

\section{Resultados}

En primer lugar se analizan los resultados a nivel descriptivo, ello se evidencia en la figura siguiente:

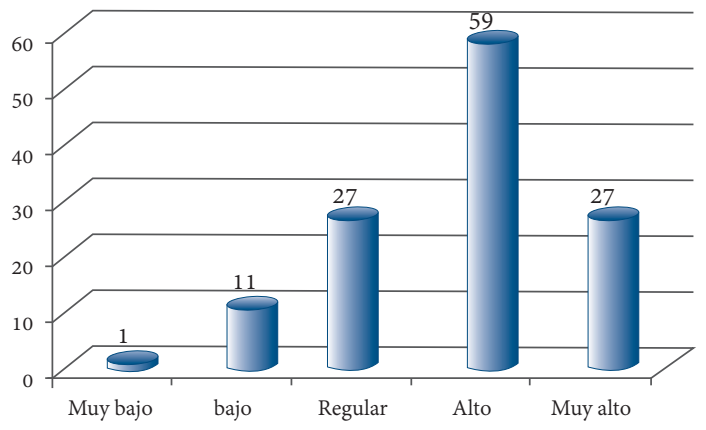

Figura 1

Nivel de competencias genéricas de los estudiantes

59 de los estudiantes tienen un nivel alto de competencias genéricas, seguido de 27 estudiantes que tienen un nivel muy alto de competencias genéricas, siendo ambos un $68.8 \%$ del total.

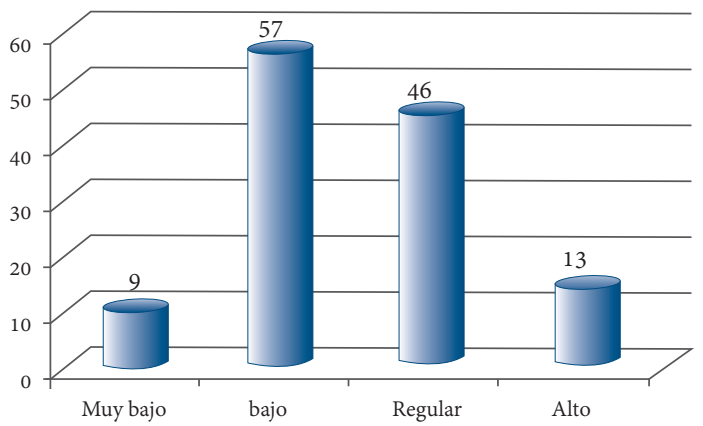

Figura 2

Nivel de estrés académico de los estudiantes
57 de los estudiantes tienen un nivel bajo de estrés académico, seguido de 46 de los estudiantes tienen un nivel regular de estrés académico, ambos grupos hacen un $82.4 \%$ del total.

A nivel inferencial, se quiere determinar la relación entre competencias genéricas y estrés académico, por ello utilizaremos el coeficiente de Pearson para determinar el grado de relación entre dichas variables. El coeficiente de correlación de Pearson ( $r$ ) es un método de correlación para variables medidas por intervalos o razón y para relaciones lineales. Se calcula a partir de las puntuaciones obtenidas en una muestra en dos variables. 


\section{Artículos Empíricos}

Tabla 1

Relación entre las variables

\begin{tabular}{ll|rr} 
& Competencias genéricas & $\begin{array}{c}\text { Competencias } \\
\text { genéricas }\end{array}$ & \multicolumn{1}{c}{$\begin{array}{c}\text { Estrés } \\
\text { académico }\end{array}$} \\
& Correlación de Pearson & 1 & $\left.-0.377^{* *}\right)$ \\
& Sig. (bilateral) & & 0.000 \\
Estrés académico & $\mathrm{N}$ & 125 & 125 \\
& Correlación de Pearson & $-0.377(* *)$ & 1 \\
& Sig. (bilateral) & 0.000 & 125 \\
\hline
\end{tabular}

${ }^{* *}$ La correlación es significativa al nivel 0,01 (bilateral).

De acuerdo a la tabla mostrada, el coeficiente de correlación de Pearson es $r=-0.377$, entonces, el grado de correlación entre las variables competencias genéricas y estrés académico es negativa media, es decir mayor competencias genéricas menor nivel de estrés académico y a menor competencia genérica mayor nivel de estrés académico.
En cuanto a las dimensiones, se quiere determinar la relación entre competencias genéricas, sus dimensiones y estrés académico, por ello utilizaremos el coeficiente de Pearson para determinar el grado de relación entre dichas variables.

Tabla 2

Relación entre las dimensiones de variables.

\begin{tabular}{llr} 
& & Estrés académico \\
Competencias instrumentales & Correlación de Pearson & $-0.364\left(^{* *}\right)$ \\
& Sig. (bilateral) & 0.000 \\
& N & 125 \\
Competencia sistémica & Correlación de Pearson & $-0.338\left(^{* *}\right)$ \\
& Sig. (bilateral) & 0.000 \\
Competencias interpersonales & Correlación de Pearson & $-0.384\left(^{* *}\right)$ \\
& Sig. (bilateral) & 0.000 \\
\hline
\end{tabular}




\section{Artículos Empíricos}

De acuerdo a la tabla mostrada, el coeficiente de correlación de Pearson, para competencias instrumentales y estrés académico es $r=-0.364$; competencia sistémica y estrés académico el coeficiente de correlación de Pearson es $\mathrm{r}=-0.338$; competencias interpersonales y estrés académico el coeficiente de correlación de Pearson es $r=$ -0.384 entonces, para todo los casos, el grado de correlación entre las dimensiones de las dos variables es negativa media.

\section{Discusión}

Desde la perspectiva descriptiva, los datos obtenidos nos indican que 57 estudiantes tienen un nivel bajo de estrés académico, seguido de 46 estudiantes que tienen un nivel regular de estrés académico, ambos grupos hacen un $82.4 \%$ del total. Estos resultados estarían en concordancia con lo encontrado por Martín (2007), Polo, Hernández y Poza (1996) cuando manifiestan que los estudiantes universitarios presentan un nivel alto de estrés académico durante el periodo de examen, mas no durante el ciclo regular de clases, debido a que en este periodo no existe mayor presión por aprobar los cursos. Es decir, los estudiantes alcanzan mayores niveles de estrés, solo durante el periodo de evaluaciones.

59 estudiantes tienen un nivel alto de competencias genéricas, seguido de 27 estudiantes que tienen un nivel muy alto de competencias genéricas, siendo ambos un $68.8 \%$ del total. Estos hallazgos nos permite aceptar la hipótesis especifica alterna que reza, existe una relación estadísticamente significativa entre competencias genéricas $y$ nivel de estrés en estudiantes universitarios, además, se puede observar que existe un nivel alto de competencias genéricas. Los resultados se podrían explicar a partir de que los estudiantes, como menciona Coronimas (2001) atribuyen una importancia crucial, a la responsabilidad, autodirección, autoformación, planificación, organización y gestión de sus propias competencias, así mismo, atribuyen importancia a la honestidad, comunicación, persistencia y relaciones personales, para sentirse muy conformes con su estilo de vida y con los logros que obtienen cuando desarrollan estas competencias.

Por otro lado, desde el punto de vista inferencial, se encuentra que el grado de correlación entre las variables competencias genéricas y estrés académico es negativa media, es decir, que a mayor competencias genéricas del estudiante, menor nivel de estrés académico y a menor competencia genérica del estudiantes, mayor nivel de estrés académico. Este resultado nos permite aceptar la hipótesis nula que dice: no existe una relación estadísticamente significativa entre las competencias genéricas y el nivel de estrés 


\section{Artículos Empíricos}

de los estudiantes de la Facultad de Ciencias Sociales de la Universidad Nacional de Educación "Enrique Guzmán y Valle”. Como era de esperarse y tomando como referencias las investigaciones de Martín (2007), Polo, Hernández y Poza (1996), existe un nivel alto de competencia genéricas y un nivel bajo de estrés académico, se debería principalmente a que los estudiantes con altas competencias, tienen mejores estrategias para gestionar el estrés y salir adelante, mientras que los estudiantes con bajos niveles de competencias genéricas posiblemente aún no pueden gestionar el estrés y terminan por aumentar sus niveles de estrés y ser más vulnerables a la hora de hacer frente a cualquier situación problémica de la vida cotidiana.

\section{Referencias}

Cornella, A. (2010). Cómo sobrevivir a la intoxicación. En Infonomia.com, 8. Barcelona.

Coromiras, E. (2001). Competencias genéricas en la formación universitaria. Revista de Educación, 325, 299-321.

Fisher, S. (1984). Stress and perception of control. London: Lawrence Erlbaum.

\section{Conclusiones}

En general, los hallazgos reportados en esta investigación no hacen más que reflejar la vigencia aún de métodos de enseñanza tradicionales, centrada en el docente más que en el estudiante. Enseñanza que no apuesta por el desarrollo de competencias tanto cognitivas, personales y sociales, específicamente no apuesta hacia la potenciación de las capacidades formativas de seres humanos a que sean, más razonables, más críticos, más estratégicos, más metacognitivos, creativos e innovadores capaces de autogestionar sus procesos de aprendizaje personal, social y académico. En buena cuenta, estudiantes autónomos, autodidactas, creativos y con altas capacidades de toma de decisiones y solución de problemas.

Fisher, S. (1986). Stress in academic life. Mental assembly line.

Gardner, H. (1994). Estructura de la mente. La teoría de las inteligencias múltiples. México: FCE.

Hernández, J.M., Polo, A.y Pozo, C. (1991). Programa de Entrenamiento en Técnicas de Estudio Servicio de Psicología Aplicada. U.A.M. 


\section{Artículos Empíricos}

Martín, I. (2007). Estrés académico en estudiantes universitarios. Apuntes de psicología, 25(1), 87-99.

Morin, E. (2001). Los siete saberes necesarios para la educación del futuro. Barcelona: Seix Barral.

Polo, A., Hernández, J. M. y Poza, C. (1996). Evaluación del estrés académico en estudiantes universitarios. Revista Ansiedad y Estrés, 2, 159-172.

Solanes, Á., Núñez, R. y Rodríguez, J. (2008). Elaboración de un cuestionario para la evaluación de competencias genéricas en estudiantes universitarios. Apuntes de psicología, 26(1), 35-49. 